\title{
Documentation of Coalitions Can Improve the Process, But Will it Lead to Better Outcomes?
}

Craig Vanderwagen, MD, RADM, USPHS; and Chad Priest, JD, MSN, RN

$\mathrm{I}$ $\mathrm{n}$ this issue of the Journal we focus on the development of regional hospital coalitions. This program was initiated by the US Department of Health and Human Services as an improvement in the Hospital Preparedness Program and has been embraced significantly during its rather brief existence. It is early in the emergence of coalition activities, but documentation of the process is underway and even some impact data seem to be emerging if not definitive outcome data.

Among other reasons, the program was conceptualized to improve efficiency and quality in handling patient surge and transfer in disaster circumstances. One of the positives that emerged from Hurricane Katrina was the timely and medically astute transfer of pediatric oncology patients who were hospitalized for cancer treatment protocols that could only be administered in inpatient settings. Accordingly, it was critical not only that these children be evacuated from flooding hospitals, but that they go to facilities and medical staffs with the capability to continue to provide high-quality cancer care without interruption. This was accomplished through the National Cancer Institute coalition for Children's Cancer Care. It was very successful with very good clinical outcomes and minimal adverse effect. The coalition funding effort is an attempt to replicate and expand such capabilities to a wide range of facilities and locations.

In addition, over the last decade, there has been an increased focus on the goal of increasing community resiliency to disaster events. Our understanding of community disaster resilience has improved as studies in Australia and elsewhere have defined the notion of community disaster resiliency more concisely and the necessary actions needed to nurture this improved survivability in the face of severe challenges. Of critical importance to improving resiliency is the "whole of community" concept. Studies are now revealing that inclusion of multiple stakeholders in examining risks, the steps that may be taken to mitigate those risks, and preparedness planning for effective response have altered community response and recovery to disasters. This reinforces what public health practitioners have long known: that the process of implementing any public health measure (including disaster preparedness) is as important as the outcome when building resilient communities.

The regional hospital coalition concept merges potential pathways to improvements in improving both community engagement and medical surge capacity. By engaging more effectively across the community, including not only hospitals, but extended care facilities, physician practice groups, primary care clinics, and specialty care providers (such as dialysis centers), these coalitions have empowered many more community members to participate and shape risk mitigation and response for medical and public health needs.

Articles in this issue describe coalition formation, membership, economics, and challenges in managing stakeholders with potentially competing interests. The article also features impact data regarding patient movement and safety. Our understanding of coalition effectiveness is still nascent. However, the articles in this issue represent an increasingly sophisticated view of coalitions: not as mere tools to implement grant deliverables, but as important public health interventions in their own right. But much more is needed in the way of measurement of the potential best practices to ensure that the theoretical advantages are real and reproducible. We hope that this issue is the beginning of a robust examination of evidence that would support real program standards in coalition development and operation. The promise is there, but the evidence needs to be documented.

\section{About the Authors}

Martin, Blanck and Associates, Alexandria, Virginia (Dr Vanderwagen), and American Red Cross, Indiana University School of Medicine, IU Center for Law, Ethics and Applied Research in Health Information, Indianapolis, Indiana (Mr Priest)

Correspondence and reprint requests to Chad Priest, Indiana University School of Medicine, 410 West 10th Street, Suite 3100, Indianapolis, IN, USA 46202. (e-mail: cspriest@iu.edu). 